\title{
Phenolic acid concentrations in the lumbar cerebrospinal fluid of Parkinsonian patients treated with L-dopa
}

\author{
I. A. PULLAR AND JUNE M. WEDDELL \\ From the M.R.C. Brain Metabolism Unit, Department of Pharmacology, \\ University of Edinburgh \\ R. AHMED AND F. J. GILLINGHAM \\ From the Department of Surgical Neurology, University of Edinburgh
}

SUMMARY The acid metabolites of dopamine and 5-hydroxytryptamine were estimated in the lumbar cerebrospinal fluid (CSF) of Parkinsonian patients both before and during treatment with L-dopa,' the amino acid precursor of dopamine. An attempt was made to relate clinical improvement to the biochemical results. The dopamine metabolites, 3,4-dihydroxyphenylacetic acid and homovanillic acid, showed increases related to the dose of L-dopa, the increase in homovanillic acid concentration being proportionately greater than that of 3,4-dihydroxyphenylacetic acid. The concentration of the 5-hydroxytryptamine metabolite, 5-hydroxyindol-3-ylacetic acid, was unaltered by the drug. Clinical improvement, which in the early stages was evident particularly in the bradykinesia, was found to occur at doses of L-dopa greater than $1.5 \mathrm{~g} /$ day. Effective doses of L-dopa gave rise to concentrations of dopamine metabolites in the CSF which were greater than normal. Possible implications of these findings are discussed.

The existence of lower concentrations of dopamine in the caudate nucleus and substantia nigra in Parkinsonian patients than in a control group (Ehringer and Hornykiewicz, 1960; Hornykiewicz, 1962) has led to the suggestion that a change in the metabolism of this amine is associated with the syndrome. This concept is supported by the finding that the main metabolite of dopamine, homovanillic acid, is also present in reduced concentrations in the brain and in the CSF of these patients (Bernheimer and Hornykiewicz, 1964; Bernheimer, Birkmayer, Hornykiewicz, Jellinger, and Seitelberger, 1966; Guldberg, Turner, Hanieh, Ashcroft, Crawford, Perry, and Gillingham, 1967; Johansson and Roos, 1967; Guldberg, Ashcroft, Turner, and Hanieh, 1969). The level of another biogenic amine, 5hydroxytryptamine is also reduced in the brain of Parkinsonian patients (Bernheimer, Birkmayer, and Hornykiewicz, 1961) and its major metabolite, 5-hydroxyindol-3-ylacetic acid, is present in subnormal concentrations in the CSF of such patients
(Guldberg et al., 1967; Johansson and Roos, 1967; Guldberg et al., 1969).

In view of a possible aetiological significance of the lowered cerebral dopamine levels in this syndrome, a number of workers (Birkmayer and Hornykiewicz, 1961; Calne, Stern, Laurence, Sharkey, and Armitage, 1969; Cotzias, Papavasiliou, Gellene, Aronson, and Mena, 1969) have treated Parkinsonian patients with the dopamine precursor, L-3,4-dihydroxyphenylalanine (L-dopa) and have observed an improvement in akinetic symptoms in many patients.

In a therapeutic trial with oral L-dopa, Calne, Karoum, Ruthven, and Sandler (1969) studied the changes in the urinary concentrations of dopamine metabolites. However, as these authors point out, the concentrations of the dopamine metabolites in the CSF are likely to mirror the metabolism of dopa in the brain more faithfully than do urinary levels. In the present study, dopa and the acid metabolites of dopamine and 5-hydroxytryptamine 
have been estimated in the lumbar CSF of Parkinsonian patients both before and during treatment with L-dopa. An attempt has been made to correlate the biochemical data with the pre-treatment clinical assessment of the patients, and with the degree of improvement observed on administration of the drug.

\section{PATIENTS AND METHODS}

The study involved 28 patients, 16 males and 12 females. Their average age was 57 , and with the exception of one male of 37, and one female of 32, their ages lay within the range of 45 to 75 years with half of them in the middle decade. The duration of the illness ranged from three to 20 years.

Before the administration of L-dopa, treatment with anti-Parkinsonian drugs was gradually stopped and each patient was rated on a simple three point scale for rigidity, tremor, bradykinesia, speech volume, and dementia and cine recordings were made (Gillingham, Watson, Donaldson, and Naughton, 1960). L-dopa was given orally, starting at a dose of $500 \mathrm{mg}$ three times a day which was increased in gradual steps. The length of time a patient remained on any one dose level and also the final maintenance dose were determined by the tolerance of the patient to the drug. In no case was the dose increased above $8 \mathrm{~g} /$ day.

Samples of lumbar CSF were obtained from the patients who had given their permission for the lumbar puncture to be performed, after it had been explained to them that the procedure was for research and not for any diagnostic purpose. Approximately $10 \mathrm{ml}$. lumbar CSF were withdrawn from the patients before the start of treatment with L-dopa and again after they had been receiving their final maintenance dose for several days. The second sample was obtained, as far as possible, four hours after the patient received a dose of the drug. If, because of side effects, the drug administration had to be terminated, the 'on treatment' CSF sample was taken immediately after the decision was made to discontinue treatment with L-dopa. Samples of CSF were stored at $-20^{\circ} \mathrm{C}$ until the analyses were carried out within not more than seven days after the sample had been withdrawn.

The concentrations of the dopamine metabolites, 3,4-dihydroxyphenylacetic acid (DOPAC) and 4-hydroxy3-methoxyphenylacetic acid (homovanillic acid, HVA), and of the 5-hydroxytryptamine metabolite, 5-hydroxyindol-3-ylacetic acid (5-HIAA) were estimated in the CSF essentially according to the methods described by Ashcroft, Crawford, Dow, and Guldberg (1968).

The cerebrospinal fluid sample was thawed, its volume noted and diluted, if necessary, to $10 \mathrm{ml}$. with deionized distilled water. It was acidified by the addition of one drop conc. hydrochloric acid (M.A.R. reagent, B.D.H.) and saturated with sodium chloride $(4 \mathrm{~g}$ Analar). The phenolic acids were removed into ethyl acetate (Analar) by shaking the sample twice with $20 \mathrm{ml}$. of the solvent for five minutes. The ethyl acetate extracts, $19 \mathrm{ml}$. from the first extraction and $20 \mathrm{ml}$. from the second, were combined after separation from the aqueous phase had been aided by centrifugation at $2,000 \mathrm{~g}$ for five minutes.

ESTIMATION OF HVA The phenolic acids in a $10 \mathrm{ml}$. portion of the pooled ethyl acetate extracts were extracted into $1.4 \mathrm{ml}$. $0.1 \mathrm{M}$ borate buffer $p \mathrm{H} 8.6$ by shaking for five minutes followed by centrifuging to separate the phases. The HVA was estimated in a $1.0 \mathrm{ml}$. portion of the buffer phase by the fluorimetric method of Andén, Roos, and Werdinius (1963) as described by Ashcroft et al. (1968).

ESTIMATION OF DOPAC AND 5-HIAA The phenolic acids in the remaining $29 \mathrm{ml}$. of the pooled ethyl acetate extract were extracted into $2 \cdot 4 \mathrm{ml}$. $0 \cdot 1 \mathrm{M}$ phosphate buffer $p \mathbf{H ~ 7 . 4 ~ b y ~ s h a k i n g ~ f o r ~ f i v e ~ m i n u t e s ~ f o l l o w e d ~ b y ~ c e n t r i f u g - ~}$ ing to separate the phases. A $1.0 \mathrm{ml}$. portion of the buffer phase was used for the fluorometric estimation of each acid as described by Ashcroft et al. (1968).

In parallel with the samples of CSF, $10 \mathrm{ml}$. of a solution containing $200 \mathrm{ng}$ each of HVA, DOPAC, and 5-HIAA c in deionized distilled water was processed to determine recoveries of the acids through the method. Reagent 'blanks' for the correction of the estimates were measured by carrying through the procedure on a $10 \mathrm{ml}$. portion of deionized distilled water.

The use of borate and phosphate buffers instead of tr. buffer as recommended by Ashcroft et al. (1968), for the extraction of the phenolic acids from the ethyl acetafe 을 extract into aqueous medium was necessitated by t unacceptably high 'blanks' obtained with the tris buffer. The phosphate buffer was less suitable than borate ${ }^{\circ} \mathbb{D}$ the procedure for HVA estimation because the form $\Phi$ was found to cause considerable quenching of to fluorescence of the fluorophor derived from HVA.

DOPA ESTIMATION Dopa in the CSF samples was estimated by the method of Guldberg and Yates (1968) after the removal of the phenolic acids by ethyl acetate extraction.

\section{RESULTS}

For various reasons it was not possible to obtain both 'pre' and 'on-treatment' samples of CSF from all the 28 patients examined, nor did it prove possible to carry out a complete analysis on every sample. The number of samples analysed for each variable is shown in the Table.

'PRE-TREATMENT' STUDIES The estimated concen- $\frac{0}{3}$ trations of DOPAC, HVA, and 5-HIAA in the lumbar CSF of the Parkinsonian patients before treatment with L-dopa are given in the Table. $₹$ The Table also shows, for comparison, the con-응 centrations found by Guldberg et al. (1969) in control subjects.

Guldberg (1967) found a significant positiven correlation between the concentrations of HVA and 5-HIAA in CSF taken from the lateral ventricles $\tilde{O}$ 
TABLE

MEAN CONCENTRATIONS (NG/ML.) OF HOMOVANILLIC ACID (HVA) 3,4-DIHYDROXYPHENYLACETIC ACID (DOPAC) AND 5-HYDROXYINDOLACETIC ACID (5-HIAA) IN LUMBAR CSF OF PATIENTS WITH PARKINSONISM AND IN CONTROL PATIENTS

\begin{tabular}{lcc}
\hline Acids & Parkinsonian & Controls \\
\hline HVA & $19 \pm 11(29)^{*}$ & $53 \pm 37(11) \dagger$ \\
DOPAC & $6 \pm 2(23)$ & $9 \pm 1(6)$ \\
5-HIAA & $13 \pm 5(26)$ & $19 \pm 4(21) \dagger$ \\
\hline
\end{tabular}

* Mean concentration \pm standard deviation (no. of patients).

†Guldberg et al. (1969)

Differences between the two groups are statistically highly significant in respect of all three acids $(P<0.005)$.

of Parkinsonian patients, such a correlation being absent in samples from control patients. In the present study a similar correlation $(r=0.66$; $P<0.001)$ has been found for the acids in the CSF taken from the lumbar spaces of Parkinsonian patients (Fig. 1).

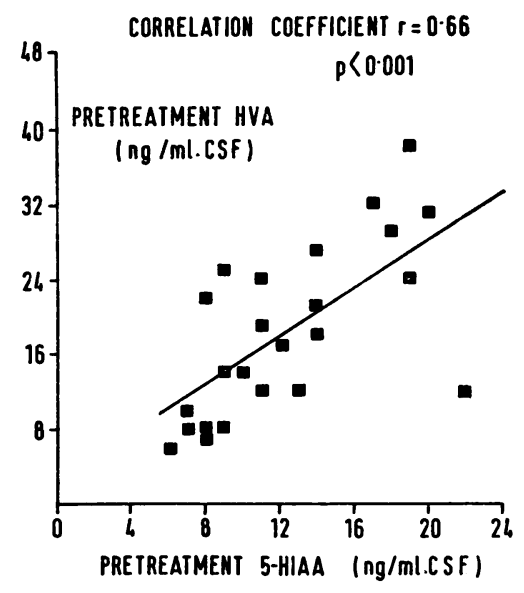

FIG. 1. Correlation between concentrations ( $\mathrm{ng} / \mathrm{ml}$. CSF) of $H V A$ and 5-HIAA in lumbar CSF of patients with Parkinsonism.

Comparison of the acid metabolite concentrations showed there was no significant difference $(P>0.05)$ between males and females or between those patients who had undergone stereotaxic surgery, and those who had not. There was no correlation $(P>0.05)$ between the concentrations of any of the phenolic acids in the CSF and the age of the patient, the duration of the illness, or severity of any of the Parkinsonian symptoms.

No dopa was detectable in the CSF of the patients before L-dopa administration.
'ON-TREATMENT' STUDIES After administration of L-dopa to the patients, the concentrations of DOPAC and HVA in the lumbar CSF increased to an extent related to the dose of L-dopa being administered at the time of lumbar fluid withdrawal (Figs. 2 and 3).

Dopa was undetectable in lumbar CSF until the dose of the amino acid was greater than $1.5 \mathrm{~g} /$ day, above which the concentration increased with increasing dose (Fig. 4). The degree of correlation between the concentration of dopa in the lumbar CSF and the dose of L-dopa was, however, low and the concentrations of HVA and DOPAC in any one sample showed a greater degree of correlation ( $r=0.83$ and 0.87 respectively) with the concentration of dopa in that sample than they did to the dose of L-dopa $(r=0.62$ and 0.58 respectively) administered to the patients at the time of lumbar fluid withdrawal (Figs. 5 and 6).

The majority of patients showed increases in the 5-HIAA concentration. The size of the increase, however, was independent of the dose of L-dopa,

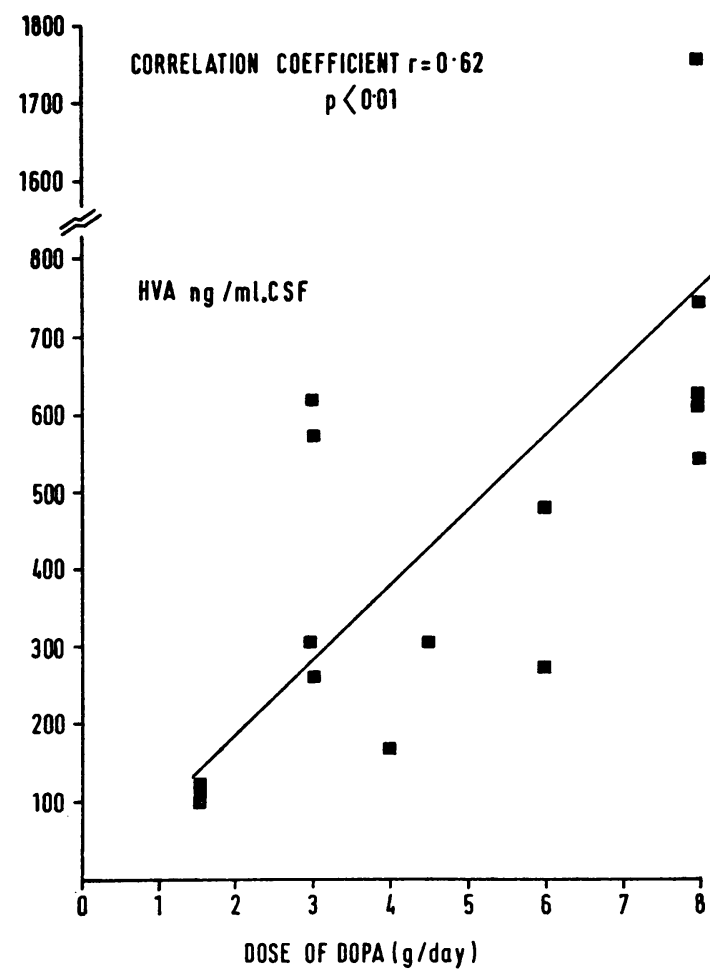

FIG. 2. Relation between the concentration (ng/ml. CSF) of $H V A$ in lumbar CSF of Parkinsonian patients and dose (g/day) of L-dopa. 


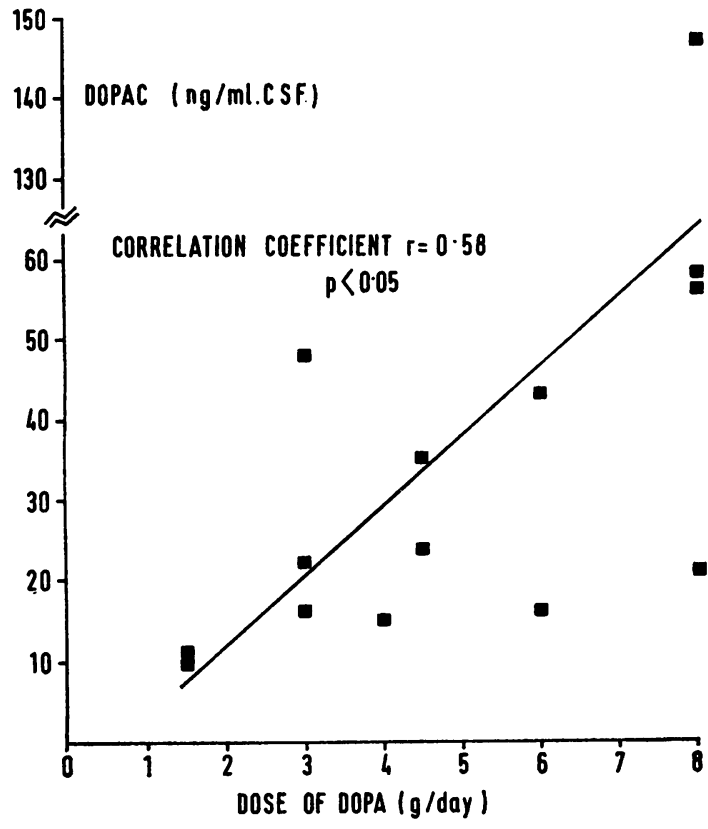

FIG. 3. Relation between the concentration (ng/ml. CSF) of DOPAC in lumbar CSF of Parkinsonian patients and dose ( $g /$ day) of $\mathrm{L}$-dopa.

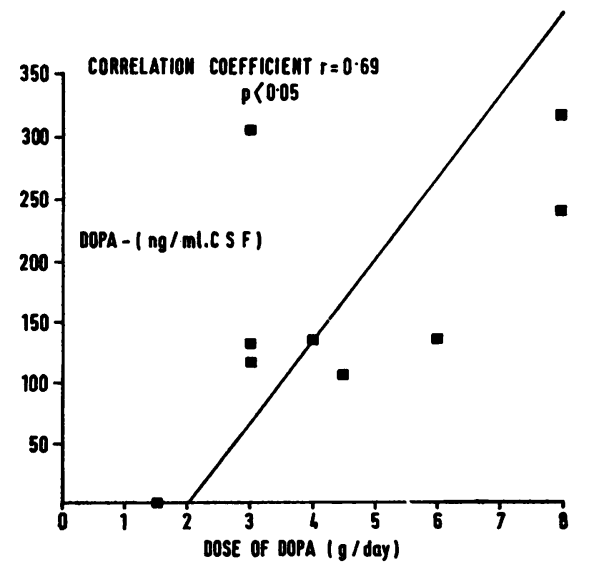

FIG. 4. Relation between concentration ( $\mathrm{ng} / \mathrm{ml}$. CSF) of dopa in lumbar CSF of Parkinsonian patients and dose ( $/$ /day) of $\mathrm{L}$-dopa.

and a paired difference analysis showed that it was not significant $(P>0.05)$.

The clinical improvement shown by these patients

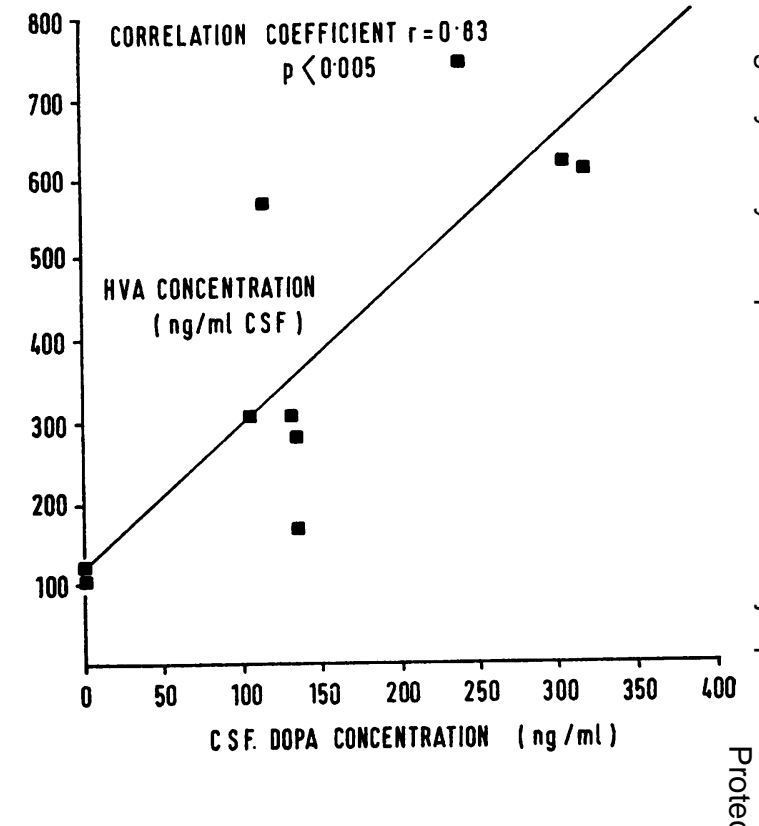

FIG. 5. Correlation between concentrations $(\mathrm{ng} / \mathrm{ml}$. CSF of $H V A$ and dopa in lumbar CSF of Parkinsonian patients treated with different doses of $\mathrm{L}-d o p a$.

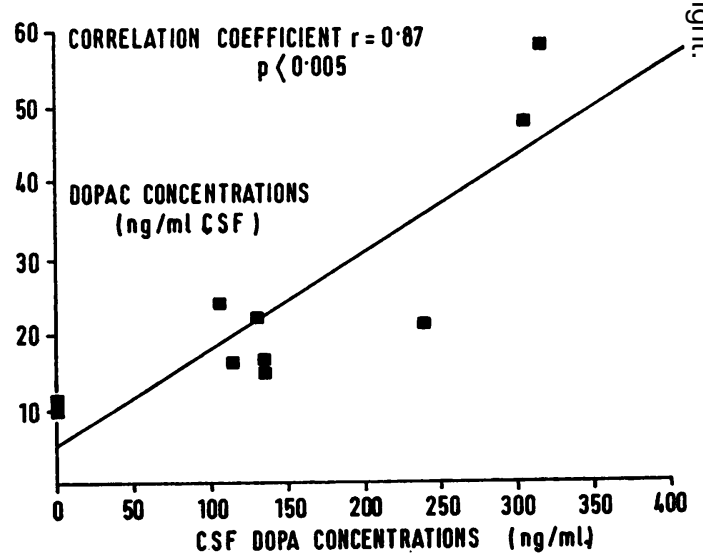

FIG. 6. Correlation between concentrations ( $\mathrm{ng} / \mathrm{ml}$. CSF) of DOPAC and dopa in lumbar CSF of Parkinsonian patients on different doses of $\mathrm{L}$-dopa.

on being given L-dopa was associated with increased concentrations of dopa, DOPAC, and HVA in the lumbar CSF as shown in Fig. 7. These obser- 


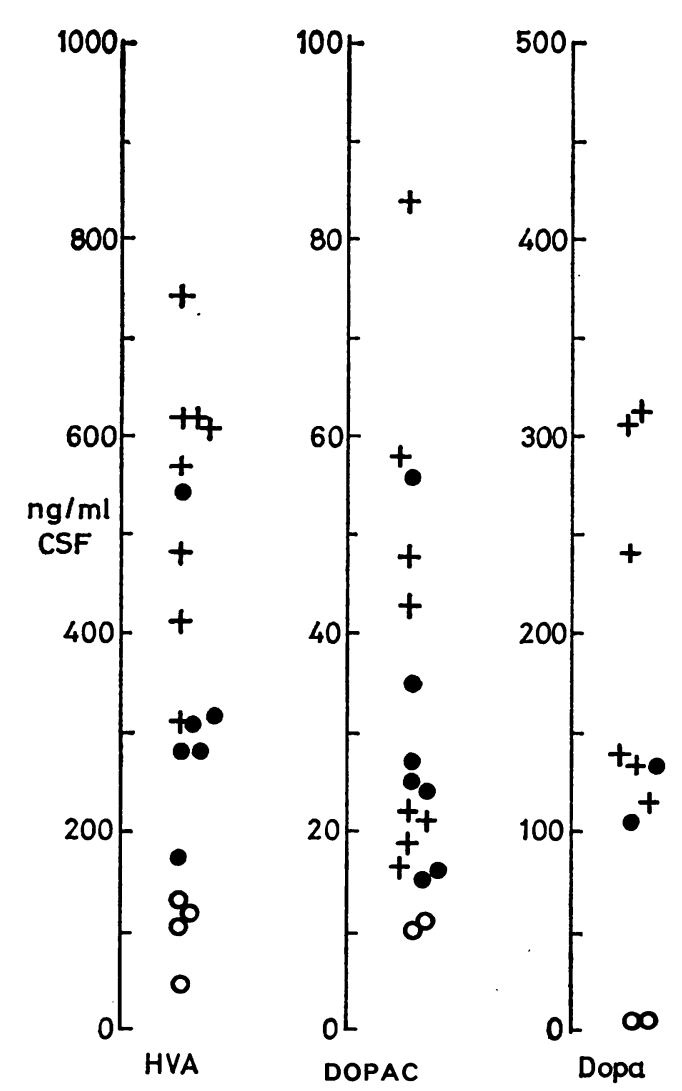

FIG. 7. Relation between concentration ( $\mathrm{ng} / \mathrm{ml}$. CSF) of $H V A, D O P A C$, and dopa in lumbar CSF of Parkinsonian patients receiving L-dopa and clinical improvement shown by these patients. $\bigcirc-$ No improvement. Moderate improvement. + - Substantial improvement.

vations are considered at greater length in the discussion. Of the 28 patients in the present study, 19 showed worthwhile improvement.

\section{DISCUSSION}

The mean concentrations of HVA and 5-HIAA found in the lumbar CSF of patients with Parkinsonism before treatment with L-dopa (Table) are similar to those recorded by Johansson and Roos (1967) and Guldberg et al. (1969) and, in comparison with the control values (Table) given by the latter workers, demonstrate the lowered levels which are a feature of the syndrome. The present study has extended these observations to include DOPAC which also shows a significantly lower concentration in the CSF of Parkinsonian patients (Table). These findings are consistent with those of Ehringer and Horny- kiewicz (1961) and Hornykiewicz (1962) of reduced dopamine levels in the caudate nucleus and substantia nigra of Parkinsonian patients, and of Bernheimer et al. (1961) of a reduction of 5hydroxytryptamine in several areas of Parkinsonian brain.

The correlation between the concentrations of HVA and 5-HIAA observed by Guldberg (1967) in the ventricular CSF, and by ourselves in the lumbar CSF of patients with Parkinsonism is not fully understood, but may reflect the progressive nature of the disease; the brain levels of dopamine and 5-hydroxytryptamine being reduced in step. There was, however, no significant correlation between the concentration of HVA, DOPAC, or 5-HIAA in the lumbar CSF of the patients and the length of time they had shown Parkinsonian symptoms. Although Parkinsonism is progressive, the rate of deterioration varies between individuals and patients who have had the disease for the same length of time may show varying severity of symptoms. There was, however, no relation between the severity of the symptoms shown by the patients and the concentration of any of the phenolic acids in the CSF.

There may exist in Parkinsonism an imbalance between the activity of dopaminergic and cholinergic systems in brain (Barbeau, 1962). This suggestion is supported by the finding that Parkinsonian-like symptoms can be produced by substances such as reserpine and chlorpromazine (May and Voegele, 1956) which are known to affect the activity, release, or metabolism of dopamine and by substances such as physostigmine which facilitate cholinergic transmission (Duvoisin, 1967). Further evidence is provided by a consideration of the methods available for treatment of the syndrome, suppression of the cholinergic system being achieved with anticholinergic anti-Parkinsonian agents and probably also by surgery. Conversely, stimulation of dopaminergic systems may be possible by administration of L-dopa leading to an increase in brain dopamine concentrations (Poirier, Singh, Sourkes, and Boucher, 1967).

Evidence that an increased cerebral synthesis of dopamine has resulted from L-dopa administration and the relationship of this increase to clinical state has been sought in the present investigation. On administration of $\mathrm{L}$-dopa the concentrations of HVA and DOPAC in the lumbar CSF increased. The rise in HVA concentration was substantially greater than that of DOPAC, contrasting with the findings of Calne et al. (1969) of an increase in urinary DOPAC concentration after L-dopa equal to or greater than the increase in HVA.

Attempts to relate the CSF levels of HVA and 
DOPAC to clinical improvement must at best remain superficial, since clinical considerations preclude manipulation of the experimental situation such as maintenance on subeffective dosage and multiple CSF sampling which would be necessary to complete the correlation profiles. However, within the limitations imposed in the present study the following observations may be made. Two patients receiving $1.5 \mathrm{~g} \mathrm{~L}$-dopa/day failed to show any clinical improvement in spite of the fact that the DOPAC and HVA concentrations in the lumbar CSF had risen to within the normal physiological range; $11 \mathrm{ng}$ and 10 $\mathrm{ng} / \mathrm{ml}$. for DOPAC and $103 \mathrm{ng}$ and $116 \mathrm{ng} / \mathrm{ml}$. for HVA. At this stage, however, no dopa was detectable in CSF. The remaining patients on doses of $3 \mathrm{~g}$ or more of $\mathrm{L}$-dopa/day showed clinical improvement associated with CSF DOPAC levels in excess of $15 \mathrm{ng} / \mathrm{ml}$. and CSF HVA levels in excess of $250 \mathrm{ng} / \mathrm{ml}$. Both these values deviate from the mean control level by six standard deviations. In excess of these critical values the degree of clinical improvement did not relate to the levels of the two acids in the CSF.

In explanation of the observation that the CSF levels of DOPAC and HVA require to reach higher than normal levels before obvious improvement in clinical status becomes apparent, it is probable that only a very small proportion of these metabolites arises from the turnover of intraneuronal dopamine derived from the administered L-dopa. Extraneuronal dopamine formation in the brains of animals treated with dopa has been demonstrated histochemically (Bertler, Falck, and Rosengren, 1963).

In addition to a different cellular site of formation of dopamine and its metabolites, there is the possibility of different metabolic routes of formation of the acids. For instance, HVA could be derived theoretically from dopa through oxidative deamination of 3-O-methyldopamine formed by decarboxylation of 3-O-methyl dopa. This pathway would bypass the formation of the biologically active amine, dopamine, in the production of HVA. The Omethylated amino acid and HVA were the only labelled metabolites found by Pletscher, Bartholini, and Tissot (1967) in the CSF of human subjects given $\mathrm{L}-\left({ }^{14} \mathrm{C}\right)$ dopa intravenously. However, 3-Omethyl dopa has been reported to be a very poor substrate for dopa decarboxylase (Ferrini and Glasser, 1964) and the extensive formation of HVA via the intermediary formation of 3-O-methyl dopa seems, on present evidence, unlikely.

It seems that L-dopa, even when it has penetrated into the general circulation after oral administration, is faced with a number of extraneuronal barriersfor example, the walls of the cerebral capillaries- or glial cells each one capable of metabolizing it before it can reach the site of neuronal dopamine formation. Estimations of HVA and DOPAC in CSF after L-dopa loading will fail to separate the contribution to the acid levels from the extraneuronal and neuronal sites. However, such estimations may still have a value in providing an index of total central nervous system metabolism as distinct from whole body metabolism.

While, to date, we have concentrated on the hypothesis that improvement in symptoms after L-dopa administration is related to stimulation of dopamine metabolism, there are other possibilitiesfor example, stimulated turnover along the noradrenaline pathway. Further studies including the simultaneous estimation of the glycol metabolites of noradrenaline and the acid metabolites of dopamine are called for with attempts to relate their levels to the symptoms present, and to the improvement produced by drug therapy.

We thank Dr. G. W. Ashcroft and Dr. T. B. B. Crawford for their invaluable help and advice in this work.

\section{REFERENCES}

Andén, N.-E., Roos, B.-E., and Werdinius, B. (1963) On the occurrence of homovanillic acid in brain ande cerebrospinal fluid and its determination by a fluorometric method. Life Sci., 2, 448-458.

Ashcroft, G. W., Crawford, T. B. B., Dow, R. C., and Guld berg, H. C. (1968). Homovanillic acid, 3,4-dihydroxy phenylacetic acid and 5-hydroxyindol-3-ylacetic acid in serial samples of cerebrospinal fluid from the laterado ventricle of the dog. Brit. J. Pharmacol., 33, 441-456.

Barbeau, A. (1962). The pathogenesis of Parkinson's disease. A new hypothesis. Canad. med. Ass. J., 87, 802-807.

Bernheimer, H., Birkmayer, W., and Hornykiewicz, O. (1961). Verteilung des 5-Hydroxytryptamins (Serotonin) im Gehirn des Menschen und sein Verhalten bei Patienten mit Parkinson-Syndrom. Klin. Wschr., 39, 1056-1059.

Bernheimer, H., Birkmayer, W., Hornykiewicz, O., Jellinger, K., and Seitelberger, F. (1966). Zur Differenzierung des Parkinson-Syndroms: Biochemisch-neurohistologische Vergleichs-untersuchungen. Proc. 8th Int. Congress of Neurology, Vienna 1965. Wiener Medizinische Akademie.

Bernheimer, H., and Hornykiewicz, O. (1964). Das Verhalten des Dopamin-Metaboliten Homovanillinsäure im Gehirn von normalen und Parkinson-kranken Menschen. Arch. exp. Path. Pharmak., 247, 305-306.

Bertler, $\AA$., Falck, B., and Rosengren, E. (1963). The direct demonstration of a barrier mechanism in the brain capillaries. Acta. pharmacol. (Kbh.), 20, 317-321.

Birkmayer, W., and Hornykiewicz, O. (1961). Der L3,4-Dioxyphenylalanin (=DOPA)-Effekt bei der Parkinson-Akinese. Wein. klin. Wschr., 73, 787-788.

Calne, D. B., Karoum, F., Ruthven, C. R. J., and Sandler, M. (1969). The metabolism of orally administered LDOPA in Parkinsonism. Brit. J. Pharmacol., 37, 57-68.

Calne, D. B., Stern, G. M., Laurence, D. R., Sharkey, J., and Armitage, P. (1969). L-DOPA in post-encephalitic parkinsonism. Lancet, 1, 744-746.

Cotzias, G. C. Papavasiliou, P. S., Gellene, Rosemary, Aronson, R. B., and Mena, I. (1969). Long term effects 
of DOPA on parkinsonism: A proposal. pp. 178-181. Third Symposium on Parkinson's Disease, Edinburgh 1968, Livingstone.

Duvoisin, R.C. (1967). Cholinergic-anticholinergic antagonism in parkinsonism. Arch. Neurol. (Chic.), 17, 124-1j6.

Ehringer, H., and Hornykiewicz, O. (1960). Verteilung von Noradrenalin und Dopamin (3-Hydroxytyramin) im Gehirn des Menschen und ihr Verhalten bei Erkrankungen des extrapyramidalen Systems. Klin. Wschr., 38, 1236-1239.

Ferrini, R., and Glasser, A. (1964). In-vitro decarboxylation of new phenylalanine derivatives. Biochem. Pharmacol., 13, 798-801.

Gillingham, F. J., Watson, W. S., Donaldson, A. A., and Naughton, J. A. L. (1960). The surgical treatment of parkinsonism. Brit. med. J., 2, 1395-1402.

Guldberg, H. C. (1967). Studies on the metabolism of cerebral catecholamines and 5-hydroxytryptamine and the influence of drugs thereon. Ph.D. Thesis, University of Edinburgh.

Guldberg, H. C., Ashcroft, G. W., Turner, J. W., and Hanieh, A. (1969). The use of cerebrospinal fluid in the study of dopamine and 5-hydroxytryptamine metabolism in parkinsonism. pp. 5C-54. Third Symposium cn Parkinson's Disease, Edinburgh, 1968, Livingstone, Edinburgh.

Guldberg, H. C., Turner, J. W., Hanieh, A., Ashcroft, G. W., Crawford, T. B. B., Perry, W. L. M., and Gillingham, F. J. (1967). On the occurrence of homovanillic acid and 5-hydroxyindol-3-ylacetic acid in the ventricular CSF of patients suffering from parkinsonism. Confin. neurol. (Basel.), 29, 73-77.

Guldberg, H. C., and Yates, Celia, M. (1968). Some studies of the effects of chlorpromazine, reserpine and dihydroxyphenylalanine on the concentrations of homovanillic acid, 3,4-dihydroxyphenylacetic acid and 5-hydroxyindol-3ylacetic acid in ventricular cerebrospinal fluid of the dog using the technique of serial sampling of the cerebrospinal fluid. Brit. J. Pharmacol., 33, 457-471.

Hornykiewicz, O. (1962). Dopamin (3-hydroxytyramin) im Zentralnervensystem und seine Beziehung ParkinsonSyndrom des Menschen. Dtsch. med. Wschr., 87, 18071810.

Johansson, B., and Roos, B.-E. (1967). 5-hydroxyindoleacetic and homovanillic acid levels in the cerebrospinal fluid of healthy volunteers and patients with Parkinson's syndrome. Life Sci., 6, 1449-1454.

May, R. H., and Voegele, G. E. (1956). Parkinsonian reactions following chlorpromazine and reserpine. Similar reactions in the same patients. Arch. Neurol. Psychiat. (Chic.), 75, 522-524.

Pletscher, A., Bartholini, G., and Tissot, R. (1967). Metabolic fate of $\mathrm{L}-\left({ }^{14} \mathrm{C}\right)$ DOPA in cerebrospinal fluid and blood plasma of humans. Brain Res., 4, 106-109.

Poirier, L. J., Singh, P., Sourkes, T. L., and Boucher, R. (1967). Effect of amine precursors on the concentration of striatal dopamine and serotonin in cats with and without unilateral brain stem lesions. Brain Res., 6, 654-666. 